\title{
Quark confinement to be caused by Abelian or non-Abelian dual superconductivity in the SU(3) Yang-Mills theory
}

\author{
Akihiro Shibata* \\ Computing Research Center, High Energy Accelerator Research Organization (KEK) \\ E-mail: Akihiro.Shibatadkek.jp \\ Kei-Ichi Kondo \\ Department of Physics, Graduate School of Science, Chiba University, Chiba 263-8522, Japan \\ E-mail: kondokefaculty.chiba-u.jp \\ Seikou Kato \\ Oyama National College of Technology, Oyama, Tochigi 323-0806, Japan \\ E-mail:Skatodoyama-ct.ac.jp
}

\section{Toru Shinohara}

Department of Physics, Graduate School of Science, Chiba University, Chiba 263-8522, Japan

E-mail:Sinoharadgraduate.chiba-u.jp

\begin{abstract}
The dual superconductivity is a promising mechanism for quark confinement. We have presented a new formulation of the Yang-Mills theory on the lattice that enables us to change the original non-Abelian gauge field into the new field variables such that one of them called the restricted field gives the dominant contribution to quark confinement in the gauge independent way. We have pointed out that the SU(3) Yang-Mills theory has another reformulation using new field variables (minimal option), in addition to the way adopted by Cho, Faddeev and Niemi (maximal option). In the past lattice conferences, we have shown the numerical evidences that support the non-Abelian dual superconductivity using the minimal option for the SU(3) Yang-Mills theory. This result should be compared with Abelian dual superconductivity obtained in the maximal option which is a gauge invariant extension of the conventional Abelian projection method in the maximal Abelian gauge.

In this talk, we focus on discriminating between two reformulations, i.e., maximal and minimal options of the $S U$ (3) Yang-Mills theory from the viewpoint of dual superconductivity for quark confinement. We investigate the confinement/deconfinement phase transitions at finite temperature in both options, which are compared with the original Yang-Mills theory. For this purpose, we measure the distribution of Polyakov-loops and the Polyakov-loop average, the correlation function of the Polyakov loops and the distribution of the chromoelectric flux connecting a quark and antiquark in both confinement and deconfinement phases.
\end{abstract}

34th annual International Symposium on Lattice Field Theory

24-30 July 2016

University of Southampton, UK

* Speaker. 


\section{Introduction}

The dual superconductivity is a promising mechanism for quark confinement [W]. In order to establish this picture, we have presented a new formulation of the Yang-Mills theory on the lattice that enables us to decompose the original Yang-Mills (YM) gauge link valiable $U_{x, \mu}$ into the gauge link variable $V_{x, \mu}$ corresponding to its maximal stability subgroup of the gauge group and the remainder $X_{x, \mu}, U_{x, \mu}=X_{x, \mu} V_{x, \mu}$, where the restricted field $V_{x, \mu}$ could be the dominant mode for quark confinement. (For a review see [D]). For the $S U$ (3) YM theory, we have two options of the decomposition: the minimal and maximal options. In the minimal option, especially, the maximal stability group is non-Abelian $U(2)$ and the restricted field contains the non-Abelian magnetic monopole. In the preceding works, we have shown numerical evidences of the non-Abelian dual superconductivity using the minimal option for the $S U(3)$ YM theory on a lattice: the restricted field and the extracted non-Abelian magnetic monopole dominantly reproduces the string tension in the linear potential of the $S U$ (3) YM theory [ [0], and the $S U$ (3) YM vacuum is the type I dual superconductor detected by the chromoelectric flux tube and the magnetic monopole current induced around it, which is a novel feature obtained by our simulations [6]. We have further investigated the confinement/deconfinement phase transition in view of this non-Abelian dual superconductivity

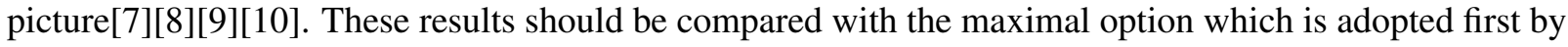
Cho, Faddeev, and Niemi [ए2]. The maximal stablity group is Abelian $U(1) \times U(1)$ and the restricted field involves only the Abelian magnetic monopole [[13][[14]. This is nothing but the gauge invariant extension of the Abelian projection in the maximal Abelian gauge[[5]][ㅈ].

In this talk, we focus on discriminating between two reformulations, i.e., maximal and minimal options of the $S U$ (3) YM theory from the viewpoint of dual superconductivity for quark confinement. For this purpose, we measure string tension for the restricted non-Abelian field of both minimal and maximal option in comparison with the string tension for the original YM field. We also investigate the dual Meissner effect by measuring the distribution of the chromoelectric flux connecting a quark and an antiquark and the induced magnetic-monopole current around the flux tube.

\section{Gauge link decompositions}

Let $U_{x, \mu}=X_{x, \mu} V_{x, \mu}$ be a decomposition of the YM link variable $U_{x, \mu}$, where the YM field and the decomposed new variables are transformed by full $S U(3)$ gauge transformation $\Omega_{x}$ such that $V_{x, \mu}$ is transformed as a gauge link variable and $X_{x, \mu}$ as a site variable [प]]:

$$
\begin{aligned}
U_{x, \mu} \longrightarrow U_{x, \mu}^{\prime} & =\Omega_{x} U_{x, \mu} \Omega_{x+\mu}^{\dagger}, \\
V_{x, \mu} \longrightarrow V_{x, \mu}^{\prime} & =\Omega_{x} V_{x, \mu} \Omega_{x+\mu}^{\dagger}, X_{x, \mu} \longrightarrow X_{x, v}^{\prime}=\Omega_{x} X_{x, \mu} \Omega_{x}^{\dagger}
\end{aligned}
$$

For the SU(3) YM theory, we have two options discriminated by its stability group, so called the minimal and maximal options.

\subsection{Minimal option}

The minimal option is obtained for the stability subgauge group $\tilde{H}=U(2)=S U(2) \times U(1) \subset S U(3)$. By introducing the color field $\mathbf{h}_{x}=\xi\left(\lambda^{8} / 2\right) \xi^{\dagger} \in S U(3) / U(2)$ with $\lambda^{8}$ being the last diagonal Gell-Mann 
matrix and $\xi$ an $S U(3)$ group element, the decomposition is given by the defining equation:

$$
\begin{aligned}
& D_{\mu}^{\varepsilon}[V] \mathbf{h}_{x}:=\frac{1}{\varepsilon}\left[V_{x, \mu} \mathbf{h}_{x+\mu}-\mathbf{h}_{x} V_{x, \mu}\right]=0, \\
& g_{x}:=e^{i 2 \pi q / 3} \exp \left(-i a_{x}^{0} \mathbf{h}_{x}-i \sum_{j=1}^{3} a_{x}^{(j)} \mathbf{u}_{x}^{(j)}\right) .
\end{aligned}
$$

Here, the variable $g_{x}$ is an undetermined parameter from Eq.(‥2a), $\mathbf{u}_{x}^{(j)}$ 's are $s u(2)$-Lie algebra valued, and $q$ has an integer value. These defining equations can be solved exactly, and the solution is given by

$$
\begin{aligned}
X_{x, \mu} & =\widehat{L}_{x, \mu}^{\dagger} \operatorname{det}\left(\widehat{L}_{x, \mu}\right)^{1 / 3} g_{x}^{-1}, \quad V_{x, \mu}=X_{x, \mu}^{\dagger} U_{x, \mu}, \\
\widehat{L}_{x, \mu} & :=\left(L_{x, \mu} L_{x, \mu}^{\dagger}\right)^{-1 / 2} L_{x, \mu}, \\
L_{x, \mu} & :=\frac{5}{3} \mathbf{1}+\frac{2}{\sqrt{3}}\left(\mathbf{h}_{x}+U_{x, \mu} \mathbf{h}_{x+\mu} U_{x, \mu}^{\dagger}\right)+8 \mathbf{h}_{x} U_{x, \mu} \mathbf{h}_{x+\mu} U_{x, \mu}^{\dagger} .
\end{aligned}
$$

Note that the above defining equations correspond to the continuum version: $D_{\mu}[\mathscr{V}] \mathbf{h}(x)=0$ and $\operatorname{tr}\left(\mathbf{h}(x) \mathscr{X}_{\mu}(x)\right)$ $=0$, respectively. In the naive continuum limit, we have reproduced the decomposition $\mathbf{A}_{\mu}(x)=\mathbf{V}_{\mu}(x)+$ $\mathbf{X}_{\mu}(x)$ in the continuum theory [B] .

The decomposition is uniquely obtained as the solution ([2.3) of Eqs.([2.2), if color fields $\left\{\mathbf{h}_{x}\right\}$ are obtained. To determine the configuration of color fields, we use the reduction condition to formulate the new theory written by new variables $\left(X_{x, \mu}, V_{x, \mu}\right)$ which is equipollent to the original YM theory. Here, we use the reduction functional:

$$
F_{\text {red }}\left[\mathbf{h}_{x}\right]=\sum_{x, \mu} \operatorname{tr}\left\{\left(D_{\mu}^{\varepsilon}\left[U_{x, \mu}\right] \mathbf{h}_{x}\right)^{\dagger}\left(D_{\mu}^{\varepsilon}\left[U_{x, \mu}\right] \mathbf{h}_{x}\right)\right\},
$$

and then color fields $\left\{\mathbf{h}_{x}\right\}$ are obtained by minimizing the functional (R.4).

\subsection{Maximal option}

The maximal option is obtained for the stability subgroup of the maximal tarus group $\tilde{H}=U(1) \times$ $U(1) \subset S U(3)$, and the resulting decomposition is the gauge-invariant extension of the Abelian projection in the maximal Abelian ( M A ) gauge. By introducing the color field $\mathbf{n}^{(3)}=\xi\left(\lambda^{3} / 2\right) \xi^{\dagger}, \mathbf{n}^{(8)}=$ $\xi\left(\lambda^{8} / 2\right) \xi^{\dagger} \in S U(3) / U(2)$ with $\lambda^{3}, \lambda^{8}$ being the two diagonal Gell-Mann matrices and $\xi$ an $S U(3)$ group element, the decomposition is given by the defining equation:

$$
\begin{aligned}
& D_{\mu}^{\varepsilon}[V] \mathbf{n}_{x}^{(j)}:=\frac{1}{\varepsilon}\left[V_{x, \mu} \mathbf{n}_{x+\mu}^{(j)}-\mathbf{n}_{x}^{(j)} V_{x, \mu}\right]=0 \quad j=3,8, \\
& g_{x}:=e^{i 2 \pi q / 3} \exp \left(-i a_{x}^{3} \mathbf{n}_{x}^{(3)}-i a_{x}^{(8)} \mathbf{n}_{x}^{(8)}\right) .
\end{aligned}
$$

The variable $g_{x}$ is an undetermined parameter from Eq.([2.5a), and $q$ has an integer value. These defining equations can be solved exactly, and the solution is given by

$$
\begin{aligned}
X_{x, \mu} & =\widehat{K}_{x, \mu}^{\dagger} \operatorname{det}\left(\widehat{K}_{x, \mu}\right)^{1 / 3} g_{x}^{-1}, \quad V_{x, \mu}=X_{x, \mu}^{\dagger} U_{x, \mu}, \\
\widehat{K}_{x, \mu} & :=\left(K_{x, \mu} K_{x, \mu}^{\dagger}\right)^{-1 / 2} K_{x, \mu}, \\
K_{x, \mu} & :=\mathbf{1}+6\left(\mathbf{n}_{x}^{(3)} U_{x, \mu} \mathbf{n}_{x+\mu}^{(3)} U_{x, \mu}^{\dagger}\right)+6\left(\mathbf{n}_{x}^{(8)} U_{x, \mu} \mathbf{n}_{x+\mu}^{(8)} U_{x, \mu}^{\dagger}\right)
\end{aligned}
$$

Note that the above defining equations correspond to the continuum version: $D_{\mu}[\mathscr{V}] \mathbf{n}^{(j)}(x)=0$ and $\operatorname{tr}\left(\mathbf{n}^{(j)}(x) \mathscr{X}_{\mu}(x)\right)=0$, respectively. In the naive continuum limit, we have reproduced the decomposition $\mathbf{A}_{\mu}(x)=\mathbf{V}_{\mu}(x)+\mathbf{X}_{\mu}(x)$ in the continuum theory.( See [B] [[2].) 

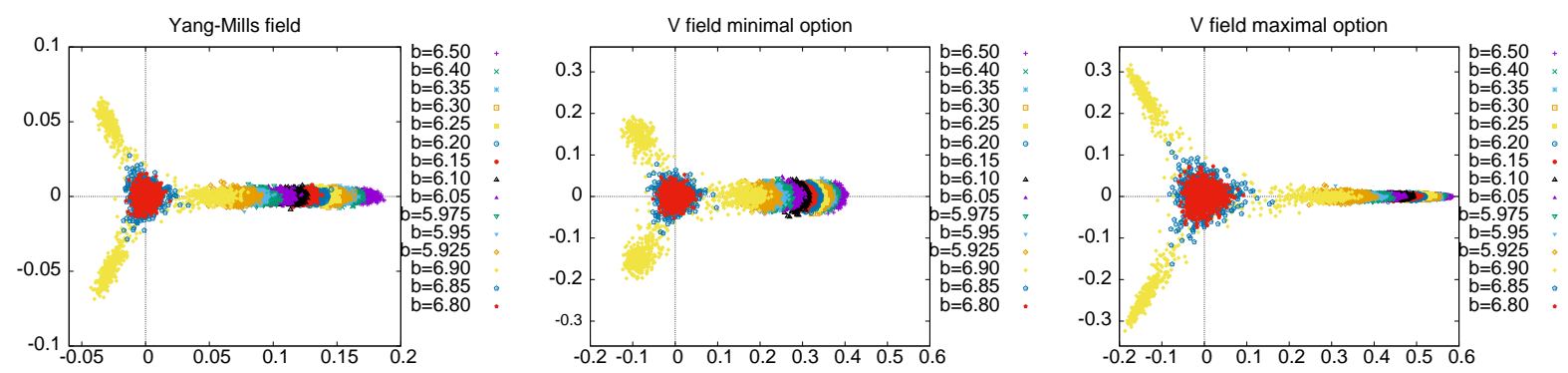

Figure 1: Distribution of the space-averaged Polyakov loops: The space-averaged Polyakov loop eq(B.2) for each configuration are plotted in the complex plane. (a) original (YM) field. (b) restricted field in the minimal option, (c) restricted field in the maximal option.

To determine the configuration of color fields, we use the reduction condition to formulate the new theory written by new variables $\left(X_{x, \mu}, V_{x, \mu}\right)$ which is equipollent to the original YM theory. Here, we use the reduction functional:

$$
F_{\mathrm{red}}\left[\mathbf{n}_{x}^{(3)}, \mathbf{n}_{x}^{(8)}\right]=\sum_{x, \mu} \sum_{j=3,8} \operatorname{tr}\left\{\left(D_{\mu}^{\varepsilon}\left[U_{x, \mu}\right] \mathbf{n}_{x}^{(j)}\right)^{\dagger}\left(D_{\mu}^{\mathcal{\varepsilon}}\left[U_{x, \mu}\right] \mathbf{n}_{x}^{(j)}\right)\right\}
$$

and then color fields $\left\{\mathbf{n}_{x}^{(3)}, \mathbf{n}_{x}^{(8)}\right\}$ are obtained by minimizing the functional (2.7). It should be noticed that the maximal option gives the gauge invariant extension of the Abelian projection in the maximal Abelian gauge.

\section{Lattice Data}

We generate the YM gauge field configurations (link variables) $\left\{U_{x, \mu}\right\}$ using the standard Wilson action on the lattice with the size of $L^{3} \times N_{T}=24^{3} \times 6$. We prepare 500 data sets at $\beta:=2 N_{c} / g^{2}\left(N_{c}=\right.$ $3)=5.8,5.9,6.0,6.1,6.2,6.3$ every 500 sweeps after 10000 thermalization. The temperature is controlled by changing the parameter $\beta$. We obtain two types of decomposed gauge link variables $U_{x, \mu}=X_{x, \mu} V_{x, \mu}$ for each gauge link by using the formula Eqs.([2.3]) and ([2.6) given in the previous section, after the colorfield configuration $\left\{\mathbf{h}_{x}\right\}$ and $\left\{\mathbf{n}_{x}^{(3)}, \mathbf{n}_{x}^{(8)}\right\}$ are obtained by solving the reduction condition as minimizing the functional ([.]) and ([2.]), respectively. In the measurement of the Wilson loop average defined below, we apply the APE smearing technique to reduce noises.

First, we investigate the distribution of a single Polyakov loop $P_{*}(\mathbf{x})$ in both options as well as the original Yang-Mills theory:

$$
P_{Y M}(\mathbf{x}):=\operatorname{tr}\left(P \prod_{t=1}^{N_{T}} U_{(\mathbf{x}, t), 4}\right), P_{\min }(\mathbf{x}):=\operatorname{tr}\left(P \prod_{t=1}^{N_{T}} V_{(\mathbf{x}, t), 4}^{(\min )}\right), P_{\max }(\mathbf{x}):=\sum_{\mathbf{x}} \operatorname{tr}\left(P \prod_{t=1}^{N_{T}} V_{(\mathbf{x}, t), 4}^{(\max )}\right),
$$

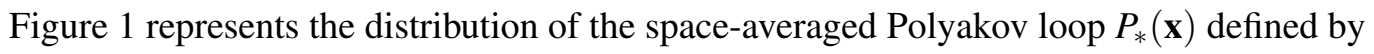

$$
P_{Y M}=\frac{1}{L^{3}} \sum_{\mathbf{x}} P_{Y M}(\mathbf{x}), \quad P_{\min }=\frac{1}{L^{3}} \sum_{\mathbf{x}} P_{\min }(\mathbf{x}), \quad P_{\max }=\frac{1}{L^{3}} \sum_{\mathbf{x}} P_{\max }(\mathbf{x}),
$$

for each configuration in the complex plane. The value of the space-averaged Polyakov loop are different among the options, but all the distributions equally refrect the expected center symmetry $Z(3)$ of $S U(3)$. 

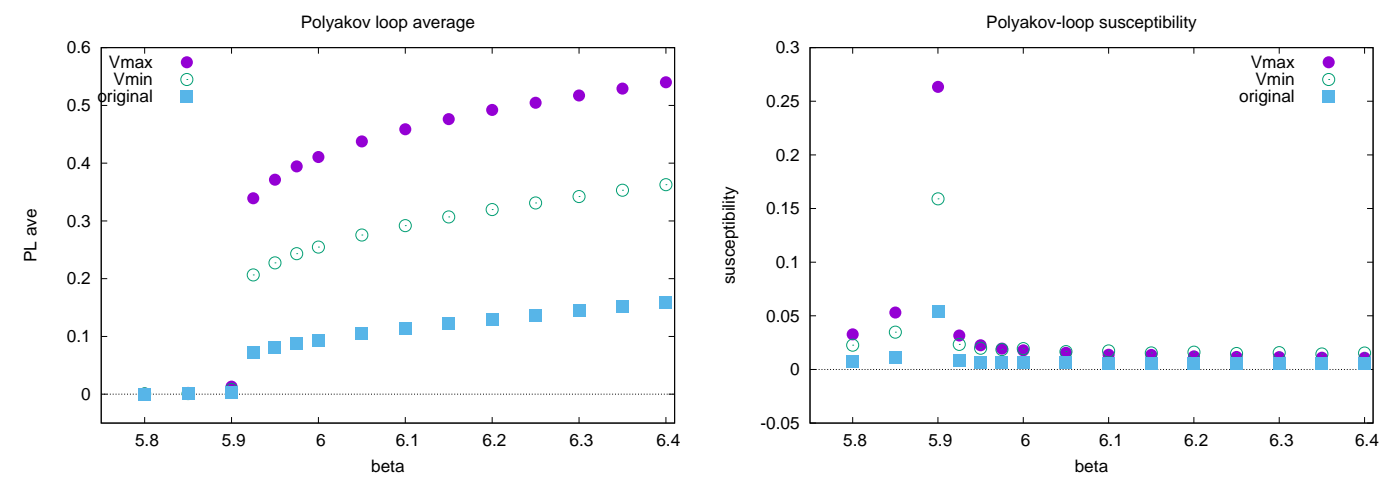

Figure 2: (Left) The combination plot of the Polyakov-loop averages for the original (YM) field, minimal option maximal option from bottom to top. (Right) The combination plot of the suseceptability of the Polyakov-loop for the original field, minimal option and maximal option.
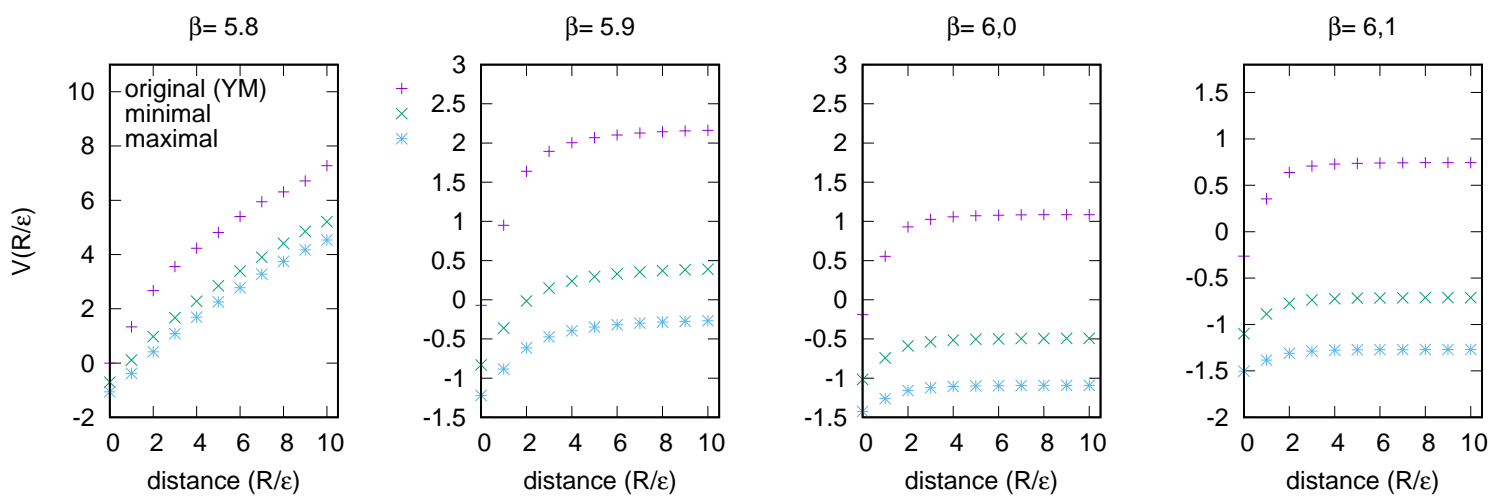

Figure 3: The two-point correlation functions of the Polyakov loops calculated from the Yang-Mills field and V-fields in the minimal and maximal options are ploted in the same panel for various values of $\beta$

The left panel of Figure $\square$ shows the Polyakov-loop average, $\left\langle P_{Y M}\right\rangle,\left\langle P_{\min }\right\rangle,\left\langle P_{\max }\right\rangle$. The Polyakov-loop average is an order parameter of the center symmetry breaking and restoration, which is the conventional order parameter of the confinement/deconfinement phase transition. The right panel of Fig.D shows the susceptibility. Fig.D shows that three Polyakov-loop average give the same critical point $\beta=5.9$. Therefore, both the minimal and maximal options reproduce the critical point in the original Yang-Mills field.

Next, we investigate the two-point correlation function of the Polyakov loops, which is related to the mixed static-potential of the singlet and adjoint composite states in the following way [ए8]]:

$$
V_{U}(r=|\mathbf{x}-\mathbf{y}|)=\log \left(\left\langle P_{U}(\mathbf{x}) P_{U}(\mathbf{y})\right\rangle\right), \quad\left\langle P_{U}(\mathbf{x}) P_{U}(\mathbf{y})\right\rangle \simeq \frac{1}{N_{c}} e^{-F(S) / T}+\frac{N_{c}^{2}-1}{N_{c}} e^{-F(A) / T} .
$$

Figure [3] shows the combination plot eq.(B.3]) for various temperature $(\beta)$. In the both options we find that the restricted field ( $V$-field) is dominant for the Polyakov loop correlation function and reproduces the static potential of the original YM theory in the long distance $r=|\mathbf{x}-\mathbf{y}|$.

Finally, we study the dual Meissner effect. For this purpose, we measure the chromo flux at finite temperature created by a quark-antiquark pair which is represented by the maximally extended Wilson loop $W$ of the $R \times T$ rectangle, i.e., the field strength of the chromo flux at the position $P$ is measured by 

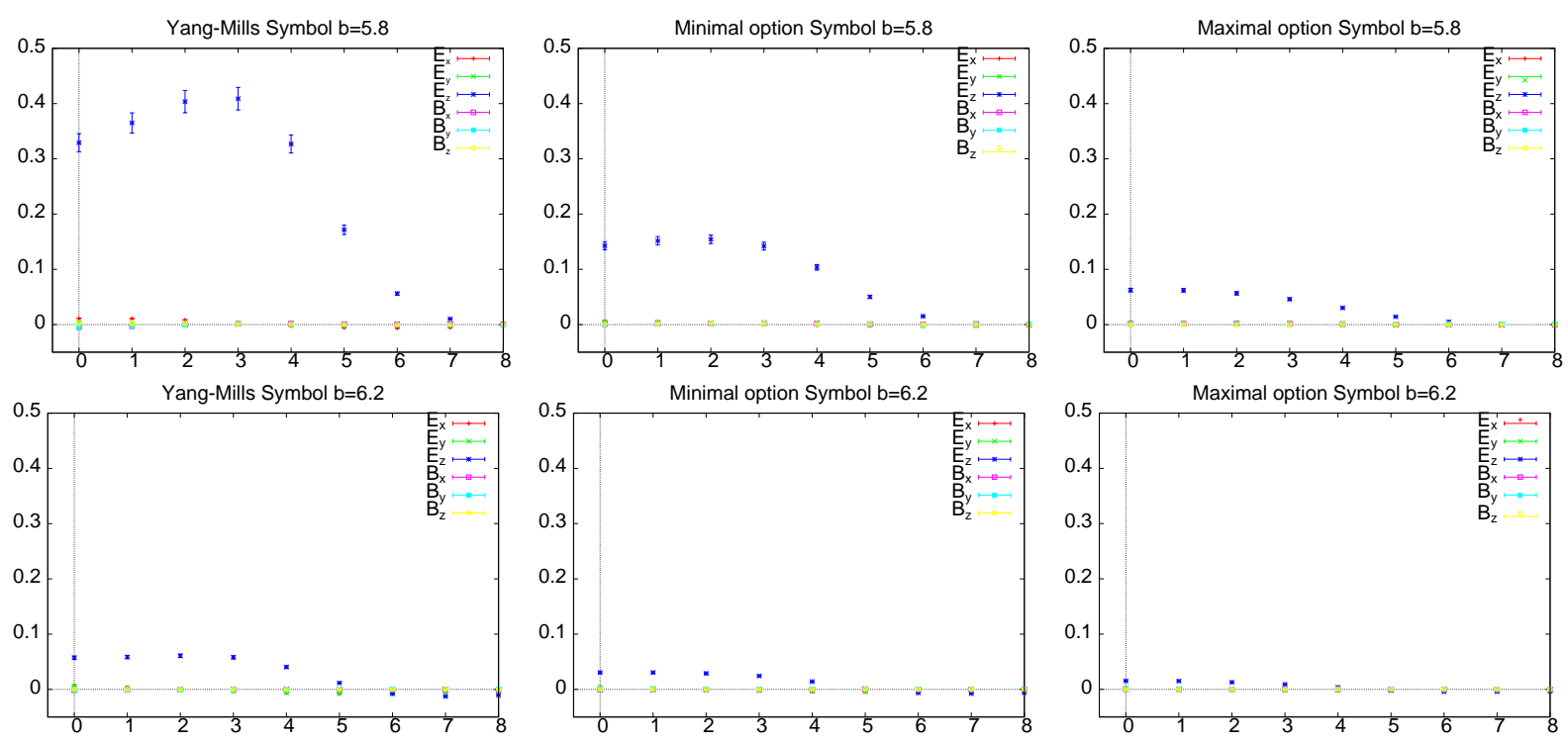

Figure 4: Chromo flux created by a pair of quark and anti-quark. The flux is measured at the point of distance y from the $1 / 3$ dividing point of quark and antiquark lying on the $\mathrm{z}$-axis. Upper and lower panels represent chromo flux in confining phase $(\beta=5.8)$ and deconfining phase $(\beta=6.2)$, respectively. Each panel in the left midle and right represents the measurement of the original (YM) field, the minimal and the maximal options, respectively.

using a plaquette variable $U_{p}$ at $P$ as the probe operator for the field strength [ए7]:

$$
\rho_{U_{P}}:=\frac{\left\langle\operatorname{tr}\left(W L U_{p} L^{\dagger}\right)\right\rangle}{\langle\operatorname{tr}(W)\rangle}-\frac{1}{3} \frac{\left\langle\operatorname{tr}\left(U_{p}\right) \operatorname{tr}(W)\right\rangle}{\langle\operatorname{tr}(W)\rangle},
$$

where $L$ is the Schwinger line connecting the source $W$ and the probe $U_{p}$ needed to obtain the gaugeinvariant result (See []$\left.^{]}\right]$). To measure the chromo flux for the restricted fields of the minimal and maximal options, we use the operator that the Schwinger line $L$ and the probe $U_{p}$ are made of the restricted fields. Figure 13 shows the preliminary measurements of the chromo flux the both options and the original field. We find that the chromo-flux tube appears in the confining phase $(\beta=5.8)$, while in the deconfining phase $(\beta=6.2$ ) the flux tube disappears, that is to say, the confinement/deconfinement phase transition is understood as the dual Meissner effect. This is the case for both options and the original YM field, although the precise profiles of the chromo flux are different option by option.

\section{Summary}

By using a new formulation of YM theory, we have investigated possible two types of the dual superconductivity at finite temperature in the $S U$ (3) YM theory, i.e., the Non-Abelian dual superconductivity as the minimal option and the maximal option to be compared with the conventional Abelian dual superconductivity. In the measurement for both maximal and minimal options as well as for the original YM field at finite temperature, we found the restricted V-field dominance for both options. The restricted fields in the both options reproduce the center symmetry breaking and restoration of the original Yang-Mills theory, and give the same critical temperature of the confining-deconfining phase transition. Then, we have investigated the dual Meissner effect and found that the chromoelectric flux tube appears in each option 
in the confining phase, but it disappears in the deconfining phase. Thus both options can be adopted as the low-energy effective description of the original Yang-Mills theory at least within the investigations presented in this talk.

\section{Acknowledgement}

This work is supported by Grant-in-Aid for Scientific Research (C) 24540252 and 15K05042 from Japan Society for the Promotion Science (JSPS), and in part by JSPS Grant-in-Aid for Scientific Research (S) 22224003. The numerical calculations are supported by the Large Scale Simulation Program No.14/15-24 (2014-2015) and No.15/16-16 (2015-2016) of High Energy Accelerator Research Organization (KEK).

\section{References}

[1] Y. Nambu, Phys. Rev. D10, 4262(1974); G. 't Hooft, in High Energy Physics, edited by A. Zichichi (Editorice Compositori, Bologna, 1975); S. Mandelstam, Phys. Report 23, 245(1976); A.M. Polyakov, Nucl. Phys. B120, 429(1977).

[2] Kei-Ichi Kondo, Seikou Kato, Akihiro Shibata and Toru Shinohara, Phys.Rept. 579 (2015) 1-226, arXiv:1409.1599 [hep-th]

[3] K.-I. Kondo, T. Murakami and T. Shinohara, Eur. Phys. J. C 42, 475 (2005); K.-I. Kondo, T. Murakami and T. Shinohara, Prog. Theor. Phys. 115, 201 (2006).; K.-I. Kondo, T. Shinohara and T. Murakami, Prog.Theor. Phys. 120, 1 (2008)

[4] K.-I. Kondo, A. Shibata, S. Kato, T. Shinohara, T. Murakami, Phys.Lett. B669 (2008) 107-118

[5] K.-I. Kondo, A. Shibata, T. Shinohara, S. Kato, Phys.Rev. D83 (2011) 114016

[6] A. Shibata, K.-I. Kondo, S. Kato and T. Shinohara, Phys.Rev. D87 (2013) 5, 05401

[7] Akihiro Shibata, Kei-Ichi Kondo, Seikou Kato, Toru Shinohara, PoS LATTICE2013 (2014)

[8] A. Shibata, K.-I. Kondo, S. Kato and T. Shinohara, PoS LATTICE2014 (2015) 340

[9] A. Shibata, K.-I. Kondo, S. Kato and T.Shinohara, KEK-PREPRINT-2015-49 CHIBA-EP-214

[10] Akihiro Shibata, Kei-Ichi Kondo, Seikou Kato, Toru Shinohara, PoS LATTICE2015 (2016) 320

[11] A. Shibata, K.-I. Kondo and T. Shinohara, Phys.Lett.B691:91-98 (2010)

[12] Y.M. Cho, Phys. Rev. D 21, 1080 (1980). Phys. Rev. D 23, 245 (1981); Y.S. Duan and M.L. Ge, Sinica Sci., 11, 1072(1979); L. Faddeev and A.J. Niemi, Phys. Rev. Lett. 82, 1624 (1999); S.V. Shabanov, Phys. Lett. B 458, 322 (1999). Phys. Lett. B 463, 263 (1999).

[13] A. Shibata, S. Kato, K.-I. Kondo, T. Shinohara and S. Ito, POS(LATTICE2007) 331

[14] Nigel Cundy, Y.M. Cho, Weonjong Lee, Jaehoon Leem, Phys.Lett. B729 192-198 (2014)

[15] Shinya Gongyo, Takumi Iritani and Hideo Suganuma, Phys.Rev. D86 (2012) 094018

[16] H. Suganuma and N. Sakumichi, Phys.Rev. D90 (2014) 11, 111501

[17] A. Di Giacomo, M. Maggiore, and S. Olejnik, Phys. Lett. B236, 199 (1990); Nucl. Phys. B347, 441 (1990).

[18] K.Yagi, T.Hatsuda and Y.Miake, Quark-Gluon Plasma (Cambridge Univ. Press, Cambridge 2005) 